\title{
Risk factors for recurrent Mycobacterium ulcerans disease after exclusive surgical treatment in an Australian cohort
}

Daniel P O'Brien

MB BS, FRACP.

Deputy Director,

Infectious Diseases

Aaron Walton MD, FRACP nfectious Diseases Physician

Andrew J Hughes MB BS, FRACP. Director, General Medicine

N Deborah Friedman MB BS, FRACP, MD Infectious Diseases Physician

Anthony McDonald MB BS, FRACS, Plastic Surgeon

Peter Callan MB BS, FRACS, Plastic Surgeon

Richard Rhadon MB BS, FRACS, Plastic Surgeon

Ian Holten MB BS, FRACS,

Plastic Surgeon

Eugene Athan MB BS, FRACP, MPH Director, Infectious Diseases

Barwon Health, Geelong, VIC

Danielo@

BarwonHealth.org.au

MJA 2013; 198: 436-439 doi: 10.5694/mjal2.11708

Case report p 443 ycobacterium ulcerans causes necrotising lesions of skin and soft tissue. The major disease burden is found in tropical climates, mainly in Africa, but cases have been reported from 33 countries worldwide. ${ }^{1}$ It is endemic in both the temperate south-eastern region and tropical areas of north-eastern Australia, where cases have recently been increasing. ${ }^{2}$

Traditionally, wide surgical excision of lesions was the recommended treatment for M. ulcerans disease, as antibiotics were felt to be ineffective. ${ }^{3,4}$ However, recurrences are common with surgical treatment alone, occurring in $16 \%-30 \%$ of cases, ${ }^{5-8}$ and patients often require multiple operations, resulting in significant morbidity, time in hospital ${ }^{6,9}$ and cost to achieve cure. ${ }^{10}$ Recently, antibiotics have been shown to be highly effective in sterilising lesions and preventing recurrences when used alone ${ }^{11-13}$ or combined with surgery. ${ }^{5}$ The World Health Organization now recommends combined antibiotic treatment for 8 weeks as first-line therapy for all M. ulcerans lesions, with surgery reserved to remove necrotic tissue, cover large skin defects and correct deformities. ${ }^{14}$

Nevertheless, especially in resource-rich settings where surgical services are readily available, exclusive surgical treatment still has a role for patients unable or unwilling to take antibiotics and those preferring the more rapid healing of small lesions that surgical excision and direct closure enables, compared with the often prolonged healing of lesions treated with antibiotics alone. ${ }^{11,13}$ In assessing a patient's suitability for exclusive surgical treatment, it is important to understand factors that increase the risk of recurrence. Previous studies have reported such risk factors, ${ }^{5-7}$ but these analyses were univariable and did not control for other potentially confounding factors

Objective: To describe risk factors for recurrence after exclusive surgical treatment of Mycobacterium ulcerans infection.

Design, setting and participants: Prospective observational cohort study of all M. ulcerans cases managed with surgery alone at Barwon Health, a tertiary referral hospital, from 1 January 1998 to 31 December 2011. A random-effects Poisson regression model was used to assess rates and associations of treatment failure.

Main outcome measures: Rates of treatment failure and rate ratios (RRs) for factors associated with treatment failure.

Results: Of 192 patients with M. ulcerans infection, 50 (26\%) had exclusive surgical treatment. Median age was 65.0 years (interquartile range [IQR], 45.5-77.7 years), and median duration of symptoms was 46 days (IQR, 26-90 days). There were 20 recurrences in 16 patients. For first lesions, the recurrence incidence rate was 41.8 ( $95 \% \mathrm{Cl}, 25.6-68.2)$ per 100 person-years, and median time to recurrence was 50 days (IQR, 30-171 days). Recurrence occurred $\leqslant 3 \mathrm{~cm}$ from the original lesion in 13 cases, and $>3 \mathrm{~cm}$ in nine. On univariable analysis, age $\geqslant 60$ years ( RR 13.84; 95\% Cl, 2.21-86.68; $P<0.01$ ), distal lesions (RR, 20.43; 95\% Cl, 1.97-212.22; $P<0.01$ ), positive histological margins (RR, 21.02; 95\% Cl, 5.51-80.26; $P<0.001$ ), immunosuppression (RR, $17.97 ; 95 \% \mathrm{Cl}$, $4.17-77.47 ; P<0.01)$ and duration of symptoms $>75$ days (RR, $10.13 ; 95 \% \mathrm{Cl}$, $1.76-58.23 ; P=0.02)$ were associated with treatment failure. On multivariable analysis, positive margins (RR, 7.72; 95\% Cl, 2.71-22.01; $P<0.001$ ) and immunosuppression ( $\mathrm{RR}, 6.45 ; 95 \% \mathrm{Cl}, 2.42-17.20 ; P=0.01$ ) remained associated with treatment failure.

Conclusions: Recurrence rates after exclusive surgical treatment of $M$. ulcerans disease in an Australian cohort are high, with increased rates associated with immunosuppression or positive histological margins.

that may have influenced outcomes. Using data from an Australian observational cohort of patients with $M$. ulcerans infection from Victoria's Bellarine Peninsula, we performed a multivariable analysis to further describe risk factors for recurrence after exclusive surgical treatment.

\section{Methods}

Data on all patients with confirmed M. ulcerans disease managed at Barwon Health were collected prospectively from 1 January 1998 to 31 December 2011. All patients who received exclusive surgery without prior antibiotics were included in the study. Patients were selected for surgery by the treating clinician's choice rather than by specified criteria. The study was approved by the Barwon Health Human Research Ethics Committee.

\section{Definitions}

An M. ulcerans case was defined as the presence of a lesion clinically suggestive of $M$. ulcerans plus any of: a culture of $M$. ulcerans from the lesion; a positive polymerase chain reaction (PCR) test result from a swab or biopsy of the lesion; or a necrotic granulomatous ulcer with the presence of acid-fast bacilli consistent with acute M. ulcerans infection on histopathological examination of an excised lesion.

The position of a lesion was defined as distal if on or below the elbow or knee. Exclusive surgical treatment was surgical excision alone, without adjunctive antibiotics. A major excision involved use of a split skin graft or vascularised skin and tissue flap to cover the defect. Positive margins were defined as the presence of granulomatous inflammation or necrotic tissue extending to one or more surgical 
excision margins on histopathological examination. Immunosuppression was defined as current treatment at any dose with immunosuppressive medication (eg, prednisolone) or presence of an active malignancy.

Treatment failure was defined as disease recurrence within 12 months of follow-up. Recurrence was defined as a new lesion appearing in the wound, locally or on another part of the body that met the $M$. ulcerans case definition. If a patient had recurrent lesions that were treated with surgery alone, it was included as a further treatment episode.

\section{Statistical analysis}

Data were collected using EpiInfo 6 (Centers for Disease Control and Prevention) and analysed using Stata 12 (StataCorp). Outcome data were censored at the time of disease recurrence, up to 12 months of follow-up from surgical treatment or until 31 October 2012.

A random-effects Poisson regression model designed to account for correlation between treatment episodes in a single patient was used to assess rates of and associations with treatment failure. Crude rate ratios for all identified variables were determined by performing univariable analyses.

An initial multivariable analysis was performed using the a priori variables of sex and age. All variables showing strong evidence of an association with treatment failure in the crude analysis $(P \leqslant 0.10)$ were then included (labelled major effect variables). The variable "duration of symptoms before diagnosis" was strongly associated with treatment failure on univariable analysis but, due to missing data, was not included in the multivariable model. All remaining variables were assessed but not included in the multivariable model as they showed evidence of multicollinearity with the major effect variables. $P$ values were determined by the likelihood ratio test. A multivariable Poisson regression model including only first episodes of treatment was also performed to test whether associations persisted when multiple episodes in individual patients were excluded.

\section{Results}

Of 192 patients with M. ulcerans infection treated at Barwon Health during the study period, 50 (26\%) had exclusive surgical treatment of an initial lesion. Baseline characteristics of patients and lesions are shown in Box 1. The median age of patients was 65.0 years (interquartile range [IQR], 45.5-77.7 years). Four patients had immunosuppression: two were taking prednisolone for polymyalgia rheumatica or eczema, and two had cancer (prostate and oesophagus). Where it was known for a patient's first lesion, the median duration of symptoms before diagnosis was 46 days (IQR, 26-90 days). No patients were lost to follow-up.

There were 58 treatment episodes: 45 patients had one treatment episode and four patients had two episodes. One patient (who was initially treated in 2002, before use of antibiotics for recurrences increased) had five surgical treatment episodes, each followed by a recurrence. Thirtyseven treatment episodes involved surgical excision and direct closure, 15 included a split skin graft, and six included a vascularised tissue flap.

There were 20 recurrences in 16 patients. The incidence rate was 41.8 (95\% CI, 25.6-68.2) per 100 personyears for first recurrences over 38.3 years' follow-up, and 48.1 (95\% CI, 31.0-74.6) per 100 person-years for all recurrences over 41.6 years' follow-up. The Kaplan-Meier curve for cumulative incidence of first recurrences is shown in Box 2. The median time to recurrence after treatment was 50 days (IQR, 30-171 days) for first lesions and 90 days (IQR, 33-171 days) for all lesions. Recurrence involved a lesion $\leqslant 3 \mathrm{~cm}$ from the original lesion in 13 cases, and $>3 \mathrm{~cm}$ in nine (two patients had recurrences both $\leqslant 3 \mathrm{~cm}$ and $>3 \mathrm{~cm}$ from the original lesion).

On univariable analysis, factors associated with treatment failure after surgery were age $\geqslant 60$ years, distal lesion position, positive margins, immunosuppression and duration of symptoms before diagnosis of $>75$ days (Box 3). On multivariable analysis, positive margins and immunosuppression remained strongly associated with treatment failure (Box 3). The
1 Baseline characteristics of the study population

\begin{tabular}{|c|c|c|}
\hline & $\begin{array}{l}\text { All patients in } \\
\text { cohort }\end{array}$ & $\begin{array}{l}\text { Patients with } \\
\text { treatment failure }\end{array}$ \\
\hline \multicolumn{3}{|c|}{ Patient characteristics } \\
\hline \multicolumn{3}{|l|}{$\operatorname{Sex}(n=50)$} \\
\hline Female & $28(56.0 \%)$ & $8(28.6 \%)$ \\
\hline Male & $22(44.0 \%)$ & $8(36.4 \%)$ \\
\hline \multicolumn{3}{|l|}{ Age $(n=50)$} \\
\hline$<60$ years & $21(42.0 \%)$ & $2(9.5 \%)$ \\
\hline$\geqslant 60$ years & $29(58.0 \%)$ & $14(48.3 \%)$ \\
\hline \multicolumn{3}{|c|}{ Diabetes $(n=50)$} \\
\hline No & $48(96.0 \%)$ & $14(29.2 \%)$ \\
\hline Yes & $2(4.0 \%)$ & $2(100.0 \%)$ \\
\hline \multicolumn{3}{|c|}{ Immunosuppression $(n=50)$} \\
\hline No & $46(92.0 \%)$ & $13(28.3 \%)$ \\
\hline Yes & $4(8.0 \%)$ & $3(75.0 \%)$ \\
\hline \multicolumn{3}{|c|}{$\begin{array}{l}\text { Duration of symptoms before } \\
\text { diagnosis }(n=44)^{*}\end{array}$} \\
\hline$\leqslant 75$ days & $32(72.7 \%)$ & $7(21.9 \%)$ \\
\hline$>75$ days & $12(27.3 \%)$ & $7(58.3 \%)$ \\
\hline \multicolumn{3}{|c|}{ Treatment episode characteristics } \\
\hline \multicolumn{3}{|c|}{ Lesion site $(n=58)$} \\
\hline Upper limb & $19(32.8 \%)$ & $6(31.6 \%)$ \\
\hline Lower limb & $35(60.3 \%)$ & $14(40.0 \%)$ \\
\hline Torso & $4(6.9 \%)$ & 0 \\
\hline Proximal & $15(25.9 \%)$ & $1(6.7 \%)$ \\
\hline Distal & $43(74.1 \%)$ & $19(44.2 \%)$ \\
\hline Not over joint & $42(72.4 \%)$ & $14(33.3 \%)$ \\
\hline Over joint & $16(27.6 \%)$ & $6(37.5 \%)$ \\
\hline \multicolumn{3}{|c|}{ Lesion type $(n=42)$} \\
\hline Ulcer & $40(95.2 \%)$ & $19(47.5 \%)$ \\
\hline Nodule & $2(4.8 \%)$ & 0 \\
\hline \multicolumn{3}{|c|}{ Major excision $(n=58)$} \\
\hline No & $37(63.8 \%)$ & $12(32.4 \%)$ \\
\hline Yes & $21(36.2 \%)$ & $8(38.1 \%)$ \\
\hline \multicolumn{3}{|c|}{ Positive margins ( $n=57)$} \\
\hline No & $37(64.9 \%)$ & $5(13.5 \%)$ \\
\hline Yes & $20(35.1 \%)$ & $15(75.0 \%)$ \\
\hline
\end{tabular}

2 Cumulative incidence of first recurrences for Mycobacterium ulcerans lesions

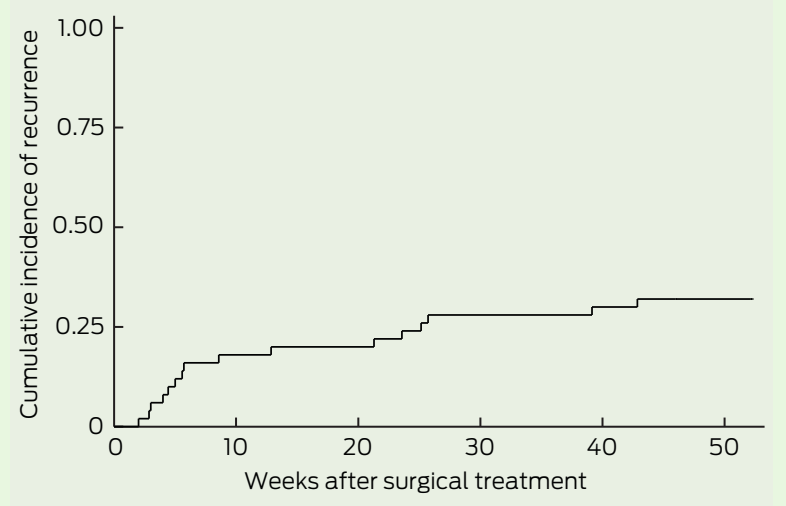


3 Poisson regression model showing adjusted and unadjusted associations between identified factors and treatment failure

\begin{tabular}{|c|c|c|c|c|c|c|c|}
\hline & $\begin{array}{l}\text { Failure } \\
\text { episodes }\end{array}$ & $\begin{array}{l}\text { Follow-up } \\
\text { (years) }\end{array}$ & $\begin{array}{c}\text { Rate per } 100 \\
\text { person-years }(95 \% \mathrm{Cl})\end{array}$ & $\begin{array}{l}\text { Crude rate ratio } \\
(95 \% \mathrm{Cl})\end{array}$ & $P$ & $\begin{array}{l}\text { Adjusted rate ratio } \\
\qquad(95 \% \mathrm{Cl})\end{array}$ & $P$ \\
\hline \multicolumn{8}{|l|}{ Sex } \\
\hline Female & 8 & 22.8 & $35.1(17.5-70.1)$ & 1 & & 1 & \\
\hline Male & 12 & 18.8 & $64.0(36.3-112.7)$ & $1.23(0.21-7.02)$ & 0.82 & $0.52(0.19-1.39)$ & 0.20 \\
\hline \multicolumn{8}{|l|}{ Age } \\
\hline$<60$ years & 2 & 19.5 & $10.2(2.6-40.9)$ & 1 & & 1 & \\
\hline$\geqslant 60$ years & 18 & 22.0 & $81.7(51.4-129.6)$ & $13.84(2.21-86.68)$ & $<0.01$ & $3.21(0.65-15.88)$ & 0.12 \\
\hline \multicolumn{8}{|l|}{ Lesion type } \\
\hline Ulcer & 19 & 24.7 & $76.8(49.0-120.4)$ & - & & - & \\
\hline Nodule & 0 & 2.0 & - & - & - & - & - \\
\hline \multicolumn{8}{|l|}{ Lesion site } \\
\hline Upper limb & 6 & 13.9 & $43.1(19.4-95.9)$ & 1 & & - & \\
\hline Lower limb & 14 & 23.7 & $59.2(35.1-99.9)$ & $1.33(0.23-7.77)$ & 0.19 & - & - \\
\hline Torso & 0 & - & - & - & & - & \\
\hline \multicolumn{8}{|c|}{ Lesion position } \\
\hline Proximal & 1 & 13.5 & $7.4(1.0-52.8)$ & 1 & & 1 & \\
\hline Distal & 19 & 28.1 & $67.6(43.1-105.9)$ & $20.43(1.97-212.22)$ & $<0.01$ & $4.49(0.58-34.51)$ & 0.07 \\
\hline \multicolumn{8}{|l|}{ Over a joint } \\
\hline No & 14 & 31.0 & $45.2(26.8-76.3)$ & 1 & & & \\
\hline Yes & 6 & 10.6 & $56.6(25.4-126.0)$ & $2.00(0.53-7.60)$ & 0.32 & - & - \\
\hline \multicolumn{8}{|c|}{ Positive margins } \\
\hline No & 5 & 32.6 & $15.3(6.4-36.9)$ & 1 & & 1 & \\
\hline Yes & 15 & 8.4 & $178.0(107.3-295.3)$ & $21.02(5.51-80.26)$ & $<0.001$ & $7.72(2.71-22.01)$ & $<0.001$ \\
\hline \multicolumn{8}{|l|}{ Major excision } \\
\hline No & 12 & 27.3 & $44.0(25.0-77.5)$ & 1 & & & \\
\hline Yes & 8 & 14.3 & $56.0(28.0-111.9)$ & $1.64(0.28-9.61)$ & 0.58 & - & - \\
\hline \multicolumn{8}{|l|}{ Diabetes } \\
\hline No & 18 & 41.2 & $43.6(27.5-69.3)$ & 1 & & & \\
\hline Yes & 2 & 0.3 & 603.7 (151.0-2413.9) & $9.94(0.43-227.8)$ & 0.13 & - & - \\
\hline \multicolumn{8}{|c|}{ Immunosuppression } \\
\hline No & 13 & 39.9 & $32.6(18.9-56.1)$ & 1 & & 1 & \\
\hline Yes & 7 & 1.7 & $416.4(198.5-873.5)$ & $17.97(4.17-77.47)$ & $<0.01$ & $6.45(2.42-17.20)$ & 0.01 \\
\hline \multicolumn{8}{|c|}{ Duration of symptoms before diagnosis } \\
\hline$\leqslant 75$ days & 7 & 27.1 & $25.8(12.3-54.2)$ & 1 & & & \\
\hline$>75$ days & 7 & 6.3 & $111.3(53.1-233.6)$ & $10.13(1.76-58.23)$ & 0.02 & - & - \\
\hline
\end{tabular}

multivariable Poisson regression model including only first episodes of treatment showed the strength of these associations persisted when multiple episodes in individual patients were excluded (data not shown).

\section{Discussion}

In our study, recurrence of $M$. ulcerans disease occurred in about a third of patients treated with surgery alone. This proportion is slightly higher than reported in studies from Africa $(17 \%-$ $22 \%)^{6,7}$ and northern Australia $(11 \%) .{ }^{15}$ In previous studies, we found adjunctive antibiotics were associated with a reduced risk of recurrence compared with surgery alone, especially if there were positive histological margins or patients had major surgery. ${ }^{5,16}$ Therefore, we recommend antibiotics as first-line therapy for $M$. ulcerans infection. However, there are patients in whom antibiotics are contraindicated, not tolerated or declined. We found $68 \%$ of patients were cured with a single surgical procedure, suggesting a role for exclusive surgical treatment as a potential alternative to antibiotics in selected cases. This study provides further prognostic information to aid decision making when considering whether surgery alone is appropriate.

We found that positive histological margins were associated with nearly an eightfold increased rate of treatment failure after surgery alone. This is likely due to incomplete excision of mycobacteria from the initial lesion, and the immune system being unable to clear them. A study from Africa similarly reported increased recurrence rates when excision was macroscopically incomplete. ${ }^{6}$ Even when excisions are performed with wide margins of macroscopically normal tissue, evidence of infection extending to excision margins is found on microscopy and PCR testing in most cases. ${ }^{17}$ Hence, we believe that histological examination of the excision margins to ensure they are free of signs of inflammation or infection is important to reduce the risk of recurrence. Nevertheless, M. ulcerans can spread subclinically from the initial lesion, including to non-contiguous body parts, ${ }^{16,18,19}$ as shown in our study by $45 \%$ of recurrences occurring $>3 \mathrm{~cm}$ from the original lesion. These distant foci will not be removed by 
wide excision of the initial lesion alone.

Studies from Africa have found increased recurrence rates in young patients $\left(<16\right.$ and $<30$ years). ${ }^{6,7}$ In our region, the disease affects mainly older adults, ${ }^{2}$ and there were not enough children in our patient population to examine this association. However, univariable analysis showed a 14-fold increased risk of recurrence in patients $\geqslant 60$ years old. An increase in the point estimate remained on multivariable analysis, but the evidence for an association was not strong. Nevertheless, it is plausible that, in older patients, a weakened immune system would allow more subclinical dissemination and thus greater risk of recurrence with surgery alone, and a study with greater patient numbers may find a stronger association. If true, this may explain the slightly higher recurrence rates seen in our older population compared with those reported from Africa. Our data suggest that until more evidence is obtained, caution should be exercised in treating patients aged $\geqslant 60$ years with surgery alone.

We found immunosuppression was associated with a sixfold increase in recurrence rates, which we believe is the first report of this association. This is biologically plausible, as T-cell immunity plays an important role in clearance of $M$. ulcerans, ${ }^{20,21}$ sometimes clearing infection in the absence of medical treatment. ${ }^{22}$ Patients with an attenuated immune response may have an increased risk of recurrence. This is supported by evidence from Mycobacterium tuberculosis treatment, where it has been shown that HIVrelated immunosuppression is a risk factor for recurrence after treatment. ${ }^{23}$

Similar to a study from the Ivory Coast, ${ }^{7}$ our univariable analysis found an increased rate of recurrence when the duration of symptoms before diagnosis was longer than 75 days. This may relate to potentially increased dissemination of mycobacteria from a lesion when present in a clinically recognisable form for longer durations.

On multivariable analysis, there was a trend toward reduced recurrence risk with proximal lesions $(P=$ $0.07)$, which may have been strength- ened with greater patient numbers. Although this association may be due to chance, other possible reasons include improved local immunity in proximal body regions; proximal body parts being more frequently covered, potentially reducing exposure to $M$. ulcerans or inhibiting its growth through higher skin temperatures; ${ }^{21}$ or wider excision margins being obtained due to easier closure of proximal wounds.

Our study has several limitations. First, it is observational and there may be other unmeasured confounders that could affect the validity of the findings. Second, the number of patients was small, affecting the power of multivariable analyses to detect weaker associations with identified variables. Third, there were no data on lesion size, so we could not measure its effect on outcomes. However, the included data on the type of surgery broadly separates small and large lesions, as small lesions are amenable to excision and direct closure, whereas larger lesions require split skin graft or vascularised flaps. Finally, missing data prevented testing the strength of the association of duration of symptoms before diagnosis in the multivariable model, thus weakening conclusions regarding its effect.

In conclusion, recurrence rates after exclusive surgical treatment of $M$. ulcerans infection in this Australian cohort are high, with increased rates associated with immunosuppression or positive histological margins on excised lesions. Our findings suggest that patients aged $\geqslant 60$ years and those who have had clinical symptoms longer than 75 days or with distal lesions may also be at increased risk of recurrent disease. Further research to validate these risk factors is recommended.

\section{Competing interests: No relevant disclosures.}

Received 21 Nov 2012, accepted 1 Apr 2013.

1 World Health Organization. Working to overcome the global impact of neglected tropical diseases: first WHO report on neglected tropical diseases. Geneva: WHO, 2010.

2 Boyd SC, Athan E, Friedman ND, et al. Epidemiology, clinical features and diagnosis of Mycobacterium ulcerans in an Australian population. Med J Aust 2012; 196: 341-344.

3 van der Werf TS, van der Graaf WT, Tappero JW, Asiedu K. Mycobacterium ulcerans infection. Lancet 1999; 354: 1013-1018.

4 Sizaire V, Nackers F, Comte E, Portaels F. Mycobacterium ulcerans infection: control, diagnosis, and treatment. Lancet Infect Dis 2006; 6: 288-296.

5 O'Brien DP, McDonald A, Callan P, et al. Successful outcomes with oral fluoroquinolones combined with rifampicin in the treatment of Mycobacterium ulcerans: an observational cohort study. PLoS Negl Trop Dis 2012; 6: el473.

6 Kibadi K, Mputu-Yamba JB, Mokassa B, et al. [Relapse after surgical treatment of Mycobacterium ulcerans infection (Buruli ulcer): study of risk factors in 84 patients in the Democratic Republic of the Congo] [French]. Med Trop (Mars) 2009; 69: 471-474.

7 Kanga JM, Kacou DE, Sangaré A, et al. [Recurrence after surgical treatment of Buruli ulcer in Cote d'Ivoire] [French]. Bull Soc Pathol Exot 2003; 96: 406-409.

8 Amofah G, Asamoah S, Afram-Gyening C. Effectiveness of excision of pre-ulcerative Buruli lesions in field situations in a rural district in Ghana. Trop Doct 1998; 28: 81-83.

9 Asiedu K, Etuaful S. Socioeconomic implications of Buruli ulcer in Ghana: a three-year review. Am J Trop Med Hyg 1998; 59: 1015-1022.

10 Pak J, O'Brien DP, Quek T, Athan E. Treatment costs of Mycobacterium ulcerans in the antibiotic era. Int Health 2012; 4: 123-127.

11 Nienhuis WA, Stienstra Y, Thompson WA, et al. Antimicrobial treatment for early, limited Mycobacterium ulcerans infection: a randomised controlled trial. Lancet 2010; 375: 664-672.

12 Chauty A, Ardant MF, Marsollier L, et al. Oral treatment for Mycobacterium ulcerans infection: results from a pilot study in Benin. Clin Infect Dis 2011; 52: 94-96.

13 Sarfo FS, Phillips R, Asiedu K, et al. Clinical efficacy of combination of rifampin and streptomycin for treatment of Mycobacterium ulcerans disease. Antimicrob Agents Chemother 2010; 54: 3678-3685.

14 World Health Organization. Treatment of Mycobacterium ulcerans disease (Buruli ulcer): guidance for health workers. Geneva: WHO, 2012.

15 Steffen CM, Smith M, McBride WJ. Mycobacterium ulcerans infection in North Queensland: the "Daintree ulcer". ANZ J Surg 2010; 80: 732-736.

16 O'Brien DP, Hughes AJ, Cheng AC, et al. Outcomes for Mycobacterium ulcerans infection with combined surgery and antibiotic therapy: findings from a south-eastern Australian case series. Med J Aust 2007; 186: 58-61.

17 Bretzel G, Siegmund V, Racz P, et al. Post-surgical assessment of excised tissue from patients with Buruli ulcer disease: progression of infection in macroscopically healthy tissue. Trop Med Int Health 2005; 10: 1199-1206.

18 O'Brien DP, Athan E, Hughes A, Johnson PD. Successful treatment of Mycobacterium ulcerans osteomyelitis with minor surgical debridement and prolonged rifampicin and ciprofloxacin therapy: a case report. J Med Case Rep 2008; 2: 123.

19 Pszolla N, Sarkar MR, Strecker W, et al. Buruli ulcer: a systemic disease. Clin Infect Dis 2003; 37: e78-e82.

20 Gooding TM, Johnson PD, Campbell DE, et al. Immune response to infection with Mycobacterium ulcerans. Infect Immun 2001; 69: 1704-1707.

21 Portaels F, Silva MT, Meyers WM. Buruli ulcer. Clin Dermatol 2009; 27: 291-305.

22 Gordon CL, Buntine JA, Hayman JA, et al. Spontaneous clearance of Mycobacterium ulcerans in a case of Buruli ulcer. PLoS Negl Trop Dis 2011; 5: el290.

23 Sonnenberg P, Murray J, Glynn JR, et al. HIV-1 and recurrence, relapse, and reinfection of tuberculosis after cure: a cohort study in South African mineworkers. Lancet 2001; 358: 1687-1693. 